\title{
ERRATA
}

\section{Erratum: Optical phonons in quantum-wire structures [JETP Lett. 67, No. 2, 120-134 (25 January 1998)]}

A. Milekhin, Yu. Pusep, Yu. Yanovskiı̆, V. PreobrazhenskiĬ, and B. Semyagin

Institute of Semiconductor Physics, 630090 Novosibirsk, Russia

Pis'ma Zh. Eksp. Teor. Fiz. 67, No. 8, 597 (25 April 1998)

[S0021-3640(98)01508-4]

PACS numbers: $63.22 .+\mathrm{m}$, 78.30.Fs, 99.10. $+\mathrm{g}$

The first two sentences of the body of the paper should read as follows:

Progress in molecular-beam epitaxy (MBE) technology has made it possible to grow perfect GaAs/AlAs superlattices (SLs) on high-index GaAs surfaces, such as (011), ${ }^{1}$ $(112),{ }^{2,3}$ and (311). ${ }^{2-4}$ The lower symmetry of these SLs as compared with SLs on (100)-oriented GaAs/AlAs leads to optical anisotropy in the plane of the SL layers. ${ }^{2-6}$

Also, Eq. (1) should read

$q_{m}=m \pi /\{(n+\delta) d\}, \quad d=a / \sqrt{11}$. 